\section{BMJ Paediatrics Open}

\title{
Comorbidities of deformational plagiocephaly in infancy: a scoping review protocol
}

\author{
Lia Charalambous (D) , ${ }^{1}$ Mijna Hadders-Algra (D) , ${ }^{2}$ Edna N. Yamasaki, ${ }^{1}$ \\ Sofia Lampropoulou, ${ }^{1,3}$
}

To cite: Charalambous L, Hadders-Algra M, N. Yamasaki E, et al. Comorbidities of deformational plagiocephaly in infancy: a scoping review protocol. BMJ Paediatrics Open 2021;5:e001113. doi:10.1136/ bmjpo-2021-001113

Received 28 March 2021 Accepted 16 May 2021

\section{Check for updates}

\section{(C) Author(s) (or their} employer(s)) 2021. Re-use permitted under CC BY-NC. No commercial re-use. See rights and permissions. Published by BMJ

${ }^{1}$ Department of Life and Health Sciences, School of Sciences and Engineering, University of Nicosia, Nicosia, Cyprus ${ }^{2}$ University of Groningen, University Medical Center Groningen, Department of Pediatrics, Division of Developmental Neurology, Groningen, The Netherlands ${ }^{3}$ Department of Physiotherapy, School of Health Rehabilitation Sciences, University of Patras, Patras, Greece

Correspondence to Lia Charalambous; charalambous.|@unic.ac.cy

\section{ABSTRACT}

Introduction Deformational plagiocephaly (DP) is one of the most common cranial shape disorders in infancy. It is characterised by unilateral flattening of the skull due to head preference to one side. The literature suggests that DP is associated with comorbidities such as developmental delay, but the nature and prevalence of the comorbid impairments are still unclear and controversial. Therefore, our scoping review (ScR) aims to explore systematically the extent and nature of literature by identifying, mapping and categorising the most relevant comorbidities of DP in children up to the age of 2 years.

Methods and analysis This protocol is based on the framework outlined by Arksey and O'Malley. A systematic search will be conducted to identify relevant full text studies from 1992 to 2021 using the databases of Cochrane, MEDLINE, Google Scholar, EMBASE, PubMed and University of Nicosia EBSCO. Two independent reviewers will screen abstracts and full articles in parallel, using specific inclusion and exclusion criteria. Specifically, this review will consider studies investigating DP and relevant comorbidities in children up to the age of 2 years of life without craniosynostosis, torticollis and any other diagnosed neurodevelopmental deficiency.

The Preferred Reporting Items for Systematic Reviews and Meta-Analyses extension for ScR Checklist will be considered for results' analysis and reporting. The results will be described in a narrative form in relation to the research question and in the context of the overall study purpose.

Ethics and dissemination Research ethics approval is not required for this ScR since data will be retrieved from publicly available studies. Dissemination activities will include research findings' submission for publication in a relevant peer-reviewed journal and presentation of the results at relevant conferences.

Registration Our protocol was registered prospectively with the Open Science Framework (https://osf.io/48am3/).

\section{INTRODUCTION}

Deformational plagiocephaly (DP) is considered to be one of the most common cranial shape disorders in infancy. ${ }^{1}$ During the last 25 years it became an issue, following the American Academy of Paediatrics (AAP) recommendation to put babies to sleep on their backs to avoid the possible occurrence

\section{What is already known on this topic?}

Deformational plagiocephaly (DP) is a common cranial disorder in infancy. The literature suggests that DP's associated comorbidities such as developmental delay, are still unclear and controversial.

\section{What this study hopes to add?}

By identifying and understanding the most relevant DP comorbidities, this scoping review may promote appropriate awareness and guidance for infants with DP acquiring early intervention.

of sudden infant death syndrome (SIDS). ${ }^{2} \mathrm{~A}$ dramatic rise in prevalence of non-synostotic DP, ranging from $5 \%$ to $48 \%$ of healthy infants, has been observed, reaching its peak at the age of 4 months and diminishing thereafter. ${ }^{134}$ However, it is still unclear why only some infants develop deformational plagiocephaly among the many infants who sleep in supine position.

DP is characterised by unilateral flattening of the skull due to head positioning preference to one side early in infancy when cranial sutures are typical and open and no craniosynostosis is present. ${ }^{5} \mathrm{DP}$ is attributed mainly to intrauterine constraints or postnatal positioning rather than to intrauterine and delivery constraints resulting in skull compression. Most likely, various intrauterine and postnatal conditions result in imbalance in muscle activity and positional preference that predispose infants to DP. ${ }^{67}$ The factors involved may be extrinsic (modifiable, environmental) or intrinsic (not modifiable, genetic) ${ }^{7}$ The skull is easily mouldable in the first months of life and consequently, application of external force can lead to deformity of the cerebral cranium as well as of the viscerocranium. ${ }^{8}$ 
Additionally, literature suggests that DP is associated with being a first-born child, boys are more affected than girls, and the right side of the cranium is generally more affected than the left side. ${ }^{9}$ Furthermore, van Vlimmeren et al suggested that specific nursing habits, positional preference when sleeping, tummy time when awake $<3$ times per day and slow achievement of motor milestones are associated with the development of DP. ${ }^{6}$

The factors affecting or associating with DP are still under investigation and this leads to a grey zone in the literature, regarding the comorbidities associated with DP. Currently, reports vary on the comorbidities of DP, for instance, whether facial deformation, visual field issues and ocular problems, asymmetry in motor behaviour, muscle tone impairments and behavioural problems are associated with DP. The conflicting results may be attributed to the age of the children, the severity of DP and the interval that passed since the initial diagnosis of DP was made. ${ }^{810-12}$ Thus, as Littlefield and Kelly pointed out in 2004, it is debated whether children with DP present with functional or developmental comorbidities before the end of the second year of life (when the cranial bones have interlocked at the sutures), and if so, whether these problems are associated with developmental impairments later in childhood. ${ }^{13}$ Nowadays, the debate still remains, and it includes the question whether DP is associated with developmental delay (as a comorbidity) and whether a potentially associated developmental delay is a temporary or a persistent problem. ${ }^{11}$

A preliminary literature search for reviews on the topic showed that most studies on DP addressed (1) various forms of intervention ${ }^{1012} 14$ or (2) aetiology and determinants of $\mathrm{DP}^{1516}$ without addressing any related comorbidities. Only the review of Martiniuk et al explored the comorbidity of developmental delay. ${ }^{17}$ This review suggested that DP may be associated with developmental delay and that infants with DP should be closely monitored. However, the reported association between developmental delay and DP should be considered with caution because included studies in the review were heterogeneous with respect to (1) the nature of the population studied (eg, general population or at-risk group; for instance, Hutchison et $^{1 l^{11}}$ included only children from higher social classes), (2) the age of onset and duration of DP, (3) the way in which DP was diagnosed and assessed (subjective clinical impression or objective measurement), (4) the age of the comorbid condition investigated (infancy only, preschool age, school age) and (5) the type of the comorbid condition (eg, global developmental delay or fine motor skills impairment or learning disorders at preschool age, ${ }^{18}$ at school age ${ }^{19}$ ).

Clarity about comorbidity of DP at early age (before the age of 2years) is urgently needed. Only when we know the comorbid pattern of infants with DP we can provide them with adequate developmental care. In this respect, it is good to realise that the ongoing debate about the potential comorbidity of DP seems to lead to increased number of referrals for screening for DP and intervention. ${ }^{17}$ The main reasons for infants with DP to receive intervention are cosmetic grounds and the fear for developmental delay. Both reasons may increase the level of concern in the parents. At present, it is hard to advise parents properly as so little is known about the associated comorbidities of DP. ${ }^{20}$ Only when we know which infants with DP are at increased risk of short-term and long-term comorbidities we will be able to develop early and more focused intervention services. Such tailor-made intervention may promote the child's development and decrease the family's level of concern and society's financial cost.

Summarising the above, no review exists that evaluated in a standardised way the comorbidities associated with DP occurring until the age of 2 years, when the bones will interlock at the sutures and no more cranial morphological changes are expected. ${ }^{5}$ Therefore, this scoping review $(\mathrm{ScR})$ aims to address knowledge gaps, identify, explore and map systematically the available research on the most relevant comorbidities of DP in children up to the age of 2 years of life with respect to incidence, prevalence and effect on outcomes. As no clearly agreed definition of comorbidity exists, ${ }^{21}$ we defined comorbidity of DP for the purpose of this ScR as the functional problems (developmental, ocular, auditory, etc) that coexist with DP and require additional evaluation, documentation and sometimes specific management.

\section{METHODS AND ANALYSIS}

The framework for this study protocol was developed based on the Arksey and O'Malley's ${ }^{22}$ ScR methodology. According to this framework, there are six different stages in undertaking an ScR: (1) identifying the research question; (2) identifying relevant studies; (3) selecting studies ; (4) charting the data; (5) collating, summarising and reporting the results; and (6) consulting with relevant stakeholders. Due to time constraints, the last stage will not be addressed.

\section{Stage 1: identifying the research questions}

This ScR addresses the following primary question, which is built according to Population, Concept and Context (PCC) framework recommended by the Joanna Briggs Institute (JBI) for ScR. ${ }^{23}$

1. Which comorbidities are associated with DP in children under the age of 2 years and what is their estimated prevalence at different ages as reported in the various studies?

To further elaborate the Concept and Context element of PCC, additional questions are reviewed:

2. What are the characteristics of the comorbidities?

3. Which measures are used to assess the comorbidities in children with DP and does the type of measure affect the prevalence of the comorbidity?

\section{Inclusion criteria}

The inclusion criteria are set according to the main objectives of the ScR and in a way to incorporate the PCC elements by JBI recommendations. 
Thus, regarding the Population, this review will consider studies that include infants and toddlers up to the age of 2 years old with DP without craniosynostosis. This age limit is decided because DP is a phenomenon that is observed right after birth and/or develops in the first trimester of postnatal life. In addition, it is known that the bones of the cranium have interlocked at the sutures by the end of the second year ${ }^{5}$ precluding further clear morphological changes. Furthermore, the previous literature data revealed unclear conclusions regarding comorbidities during this age period.

To better serve the objectives of the study, this review will omit studies including infants with craniosynostosis, torticollis or any other neurological or genetic disorders that are considered as specific aetiological and pathogenetic categories associated with DP. Inclusion of specific disorders would confound the associations between positional DP (our focus) and comorbidities. ${ }^{7}$

The core Concept examined by this ScR is the prevalence and characteristics of comorbidities in children with DP. Thus, studies identifying comorbidities, such as various forms of sensory impairment, motor impairments, language impairment, general developmental delay or behavioural problems will be considered for review.

Regarding the Context element of PCC and in line with its objectives, this ScR will not apply any culture, location, racial/gender or setting restrictions. Prevalence of comorbidities will be specified according to sex, geographical settings and risk groups. Furthermore, with regard to the type of evidence sources, studies considered as valid for inclusion will be observational studies, including prospective and retrospective cohort studies, case-control studies and cross-sectional studies and case series, since in observational designs, the investigator observes and records data on a group of people, with no active manipulation of exposure conditions, generating information on the relationships between exposure and disease. ${ }^{24}$ Qualitative studies and conference abstracts will be excluded because these types of evidence are not considered by the research team appropriate to serve the objectives of the review. Comments, editorials, short communications and letters addressing eligible studies for inclusion will be reviewed to identify possible agreements and/or controversies on the topic of investigation. Systematic reviews addressing other aspects of DP will be consulted in order to identify additional eligible studies for inclusion.

During the process of criteria setting, any decisions regarding process modifications will be reviewed and agreed by the research team to ensure accuracy and to guarantee that the information retrieved serves the ScR's objectives. This may be necessary during the review process as the reviewers increase their awareness and may reconsider the contents of all included sources.

\section{Stage 2: identifying relevant studies}

The ScR search is mapped by the PCC framework as recommended by JBI $(2020)^{25}$ and in congruence with the inclusion criteria. Thus, the main keywords are categorised to above elements and they are gathered with alternative keywords or synonyms for the same concept to complete each element. Thus, in line with the main research question of the present ScR, the final search terms to be used are presented in table 1.

During the primary search, the filter of publication date will be added; it will range from 1992 to April 2021. The date of 1992 is chosen as it was the year when the first AAP recommendation regarding SIDS and safe to sleep campaign was published. ${ }^{2}$ No language restrictions will be applied during initial search. The databases to be searched include MEDLINE, CINAHL, EMBASE and PubMed. Reviewers will contact authors of eligible primary studies to obtain additional information on poorly reported items, if needed.

The searching process is planned to follow a specific three-step strategy according to updated Joanna Briggs recommendations for ScR methods $(2020) .{ }^{25}$ At the first step, an initial limited search of MEDLINE and CINAHL will be undertaken to identify articles on the topic. At this stage, the search strategy will be piloted to check the appropriateness of keywords and databases with assistance from an experienced librarian. At a second stage, all identified keywords and index terms will be used to develop a full search strategy for Cochrane, Google Scholar, EMBASE, PubMed and University of Nicosia

Table 1 Search strategy

Main research question Which comorbidities are associated with deformational plagiocephaly in children under 2 years and what is their estimated incidence and prevalence as reported in the various studies?

Population infant* OR newborn OR neonate* OR baby OR babies OR full\#term OR pre\#term OR toddler

Concept

1. deformational plagiocephal* OR positional plagiocephal ${ }^{\star}$ OR "flat head syndrome" OR posterior plagiocephal* $^{*}$

AND

2. comorbidit* OR "developmental delay" OR reflux OR cry OR crying OR ocular OR problem* OR feed* OR position* OR preference OR inciden* OR "clinical sign*” OR symptom* OR addition* OR co\#occur* OR condition* OR co\#existing OR confound* OR factor* OR associat*

Context No cultural, geographical or population factors applied

Study design cohort OR cross\#sectional OR "case control” OR observational 
EBSCO databases. The electronic search algorithm will be recorded in a table for each database. The search will be completed at a third stage by identifying articles at additional sources such as the reference lists of the sources already selected from full text and/or included in the review.

\section{Stage 3: selecting studies}

The selection of the studies will be performed according to inclusion criteria prespecified which will serve to construct the PCC framework of the ScR. Sources will be selected initially by the title/abstract screening of the databases and after the duplicates are excluded, a fulltext screening will be performed by two independent reviewers.

Potentially relevant sources and their citations will be retrieved in full text. The full text of selected citations will be assessed in detail against the inclusion criteria by two reviewers. Reasons for exclusion of sources of evidence at full text that do not meet the inclusion criteria will be recorded and reported in the ScR. Any disagreements that arise between the reviewers at each stage of the selection process will be resolved through discussion, or with an additional reviewer. The results of the search and the study inclusion process will be reported in full in the final ScR and presented in a Preferred Reporting Items for Systematic Reviews and Meta-analyses extension for ScR (PRISMA-ScR) flow diagram. ${ }^{26}$ This flow chart will provide details about the search flow, source selection, duplicates removed, full-text retrieval, and any additions from third search, data extraction steps and evidence interpretation and presentation.

\section{Stage 4: charting the data}

The JBI results extraction instrument ${ }^{25}$ will be adopted and will be modified and revised as necessary during the process of extracting data from each included evidence source. Modifications will be detailed in the final ScR report. If appropriate, authors of papers will be contacted to request missing or additional data, where required.

Data will be extracted from papers included in the $\mathrm{ScR}$ by two independent reviewers as it is recommended by JBI. ${ }^{25}$ The data extracted will include specific details about: author(s), year of publication, study location, study population (sex, age, comparator group (s) if any, sample size, perinatal history, etc), design, aims/purpose of the study, DP (age of onset, criteria, duration, assessment method), types of comorbidity and ways of measurement (eg, developmental delay outcome measures), study's methodology, interventions applied (type and duration), outcomes and details and key findings that relate to the ScR questions. The charting table will be piloted before the review stage and will be continually updated throughout the process of the review. Clear publication bias, such as bias due to confounding comorbidities or non-representative samples, will be recorded and taken into account in data collation.
Stage 5: collating, summarising and reporting the results

Final results will be grouped and coded in order to assist the organisation of the report of the review findings. For example, comorbidities will be categorised into impairments (eg, bony deformations, muscle tone alteration) and activities and participation (eg, limited mobility (manipulation, locomotion, limited abilities of learning and application of knowledge)) according to the International Classification of Functioning, Disability and Health-Child and Youth version. ${ }^{27}$ Any decisions regarding process modifications will be reviewed and agreed by the research team to ensure accuracy.

Formal assessment of the quality of included studies will not be undertaken, since this ScR's aim is to provide a map of what evidence is available rather than seeking only the best available evidence to answer this particular question related to DP comorbidities in infancy. ${ }^{28}$

Specifically, the presentation of the results will be separated mainly in two categories: (1) a basic numerical account of the amount, type and distribution of studies in relation to comorbidities; and (2) a thematic analysis and concept map of the results. ${ }^{22}$ For each conceptual category (ie, key findings, methodology adopted, gaps in the research, etc), a clear explanation will be provided or it will be determined whether a more in-depth analysis is required.

A narrative summary will accompany the tabulated and/or charted results and will describe how the results relate to the review's objective and questions. The PRISMA-ScR Checklist will be taken into consideration for results' analysis and reporting. ${ }^{26}$

Contributors LC conceived the original idea and wrote the initial draft. SL, MH-A and EY provided critical feedback and helped shape the research methodology. LC and SL are responsible for investigation process specifically performing data collection and review. $\mathrm{MH}-\mathrm{A}$ will be the additional reviewer in case of disagreement. LC wrote the manuscript with support from SL and MH-A. SL and EY helped supervise the project. All authors (LC, SL, MH-A and EY) contributed to the final version of this manuscript by critical review, commentary and/or revision.

Funding The authors have not declared a specific grant for this research from any funding agency in the public, commercial or not-for-profit sectors.

Disclaimer The paper presents the view of the authors and should not be taken to be representative of the authors' institutions.

Competing interests None declared.

Patient and public involvement Patients and/or the public were not involved in the design, or conduct, or reporting, or dissemination plans of this research.

Patient consent for publication Not required.

Ethics approval As this ScR methodology involves collecting and investigating data from already published material, no ethics approval or patient and public involvement is required. The results will be disseminated through a peer-reviewed publication and national and international conferences targeting an audience of health professionals involved in early intervention of young children.

Provenance and peer review Not commissioned; externally peer reviewed.

Data availability statement Data are available in a public, open access repository. All data relevant to the study are included in the article or uploaded as supplemental information. This is an open access article which permits others to distribute and adapt content, provided the original work is properly cited, appropriate credit is given and the use is non-commercial.

Open access This is an open access article distributed in accordance with the Creative Commons Attribution Non Commercial (CC BY-NC 4.0) license, which 
permits others to distribute, remix, adapt, build upon this work non-commercially, and license their derivative works on different terms, provided the original work is properly cited, appropriate credit is given, any changes made indicated, and the use is non-commercial. See: http://creativecommons.org/licenses/by-nc/4.0/.

\section{ORCID iDs}

Lia Charalambous http://orcid.org/0000-0001-5393-6281

Mijna Hadders-Algra http://orcid.org/0000-0001-6845-5114

\section{REFERENCES}

1 Hutchison BL, Hutchison LAD, Thompson JMD, et al. Plagiocephaly and brachycephaly in the first two years of life: a prospective cohort study. Pediatrics 2004;114:970-80.

2 American Academy of Pediatrics. American Academy of pediatrics AAP Task force on infant positioning and SIDS: positioning and SIDS. Pediatrics 1992;89:1120-6.

3 Persing J, James $\mathrm{H}$, Swanson J, et al. Prevention and management of positional skull deformities in infants. Pediatrics 2003;112:199-202.

4 American Academy of Pediatrics Task Force on Sudden Infant Death Syndrome. The changing concept of sudden infant death syndrome: diagnostic coding shifts, controversies regarding the sleeping environment, and new variables to consider in reducing risk. Pediatrics 2005; 116:1245-55.

5 Ridgway EB, Weiner HL. Skull deformities. Pediatr Clin North Am 2004:51:359-87.

6 van Vlimmeren LA, van der Graaf Y, Boere-Boonekamp MM, et al. Risk factors for deformational plagiocephaly at birth and at 7 weeks of age: a prospective cohort study. Pediatrics 2007;119:e408-18.

7 Steinmann LC SS. Nonsynostotic deformational plagiocephaly: understand, screen and intervene 2014.

8 Linz C, Kunz F, Böhm H, et al. Positional skull deformities. Dtsch Arztebl Int 2017:114:535-42.

9 Hutchison BL, Thompson JMD, Mitchell EA. Determinants of nonsynostotic plagiocephaly: a case-control study. Pediatrics 2003;112:e316.

10 Xia JJ, Kennedy KA, Teichgraeber JF, et al. Nonsurgical treatment of deformational plagiocephaly: a systematic review. Arch Pediatr Adolesc Med 2008;162:719-27.

11 Hutchison BL, Stewart AW, Mitchell EA. Deformational plagiocephaly: a follow-up of head shape, parental concern and neurodevelopment at ages 3 and 4 years. Arch Dis Child 2011;96:85-90.

12 Ellwood J, Draper-Rodi J, Carnes D. The effectiveness and safety of conservative interventions for positional plagiocephaly and congenital muscular torticollis: a synthesis of systematic reviews and guidance. Chiropr Man Therap 2020;28:1-11.

13 Littlefield TR, Kelly KM. Deformational plagiocephaly: recommendations for future research. J Prosthet Orthot 2004;16:S59-62.

14 Bialocerkowski AE, Vladusic SL, Howell SM. Conservative interventions for positional plagiocephaly: a systematic review. Dev Med Child Neurol 2005;47:563-70.

15 Bialocerkowski AE, Vladusic SL, Wei Ng C. Prevalence, risk factors, and natural history of positional plagiocephaly: a systematic review. Dev Med Child Neurol 2008;50:577-86.

16 De Bock F, Braun V, Renz-Polster H. Deformational plagiocephaly in normal infants: a systematic review of causes and hypotheses. Arch Dis Child $2017 ; ; 102: 535-42.2017 / 06$.

17 Martiniuk ALC, Vujovich-Dunn C, Park M, et al. Plagiocephaly and developmental delay: a systematic review. J Dev Behav Pediatr 2017;38:67-78.

18 Steinbok P, Lam D, Singh S, et al. Long-term outcome of infants with positional occipital plagiocephaly. Child's Nervous System 2007;23:1275-83.

19 Shamji MF, Fric-Shamji EC, Merchant P, et al. Cosmetic and cognitive outcomes of positional plagiocephaly treatment. CIM 2012;35:266-70.

20 Steinbok P, Lam D, Singh S, et al. Long-term outcome of infants with positional occipital plagiocephaly. Childs Nerv Syst 2007;23:1275-83.

21 Bax M, Gillberg C. Comorbidities in developmental disorders. John Wiley \& Sons, 2010.

22 Arksey H, O'Malley L. Scoping studies: towards a methodological framework. Int J Soc Res Methodol 2005;8:19-32.

23 Peters MDJ, Godfrey C, McInerney P. Chapter 11: Scoping Reviews (2020 version). In: Aromataris E, Munn Z, eds. JBI manual for evidence synthesis, 2020.

24 Buka SL, Rosenthal SR, Lacy ME. Epidemiological study designs: traditional and novel approaches to advance life course health development research. In: Handbook of life course health development, 2018: 541-60.

25 Peters MDJ, Marnie C, Tricco AC, et al. Updated methodological guidance for the conduct of scoping reviews. JBI Evid Synth 2020;18:2119-26.

26 Tricco AC, Lillie E, Zarin W, et al. PRISMA extension for scoping reviews (PRISMA-ScR): checklist and explanation. Ann Intern Med 2018;169:467-73.

27 World Health Organization. International Classification of Functioning, Disability, and Health: Children \& Youth Version: ICFCY; 2007.

28 Munn Z, Peters MDJ, Stern C, et al. Systematic review or scoping review? guidance for authors when choosing between a systematic or scoping review approach. BMC Med Res Methodol 2018;18:143. 